Introduction: The Electronic Health Record (EHR) is now the standard means for recording and maintaining medical notes in most emergency departments. The EHR is an independent cause of physician burnout, and maintenance of the EHR may occupy 30 to $50 \%$ of clinical time. There are software solutions available, but they are connected to fixed, expensive, distracting, and bright electronically powered computers. Scribes have been successfully trialed, but are also expensive and attached to computers on wheels. Portable digital word processors in the form of the AlphaSmart Neo is a redundant technology designed primarily for children with typing difficulties. It has recently enjoyed a resurgence in popularity among professional writers, journalists, and field researchers for the ultimate distraction-free writing experience. The Alphasmart Neo is cheap, nearly indestructible, intuitive, and requires almost no recharging. It is compatible with all software across Mac OS, Windows, and Linux. Notes are entered by the clinician or scribe, independently of computers, at the bedside, and uploaded to any software via USB cable.

Aim: To describe the introduction and impact of the AlphaSmart Neo on the EHR in emergency departments across Australia.

Methods: We will examine the role of the Alphasmart Neo in austere, low power, extreme environments with a demonstration on how to enter, maintain, and transfer an electronic health record independent of any computer or power source.

Discussion: We believe the AlphaSmart Neo is an ideal, personalized, cheap, effective, and efficient hardware solution to entering notes independent of other software and hardware. It is distraction free at the patient's bedside, resulting in better notes that the clinician enjoys writing.

Prehosp Disaster Med 2019;34(Suppl. 1):s104-s105

doi:10.1017/S1049023X19002188

\section{Basic Principles in Complex Humanitarian Emergency: A} Pilot Course

Dr. Nidaa Bajow ${ }^{1}$, Dr. yousef Alawad ${ }^{2}$, Dr. Samer Aloraifi ${ }^{1}$

1. Mohammad Bin Naif Medical Center - king Fahad Security Collage, RIYADH, Saudi Arabia

2. king Fahad Medical city - Minister Of Health, RIYADH, Saudi Arabia

Introduction: The international and national response team faces many challenges during a complex humanitarian emergency. These include difficult organization, an unprepared national disaster plan, and a disrupted political system. Previous studies showed a reactive approach in earlier disasters in Saudi Arabia and the need for greater involvement of health professionals in disaster management. As a result, several medical education and training institutes began to introduce courses which were mainly about Major Incidents Response, but with less attention to Humanitarian Assistance and Disaster Relief. Aim: The course provides Basic Principles in Complex Humanitarian Emergency for healthcare providers in the kingdom and is focused on the aspect of community awareness for disaster and humanitarian relief.

Methods: The interactive competencies-based course in Basic Principles of Complex Humanitarian Emergency was implemented. The course was designed by 5 experts in disaster medicine and humanitarian relief and was piloted over five days at officers club of Minister of Interior in Riyadh, sponsored by King Fahd Security College. The participants $(n=30)$ were from different health disciplines. They completed the pre- and posttests and presented three pilot workshops for disaster community awareness.

Results: The overall scores were $44.19 \%$ for the pre-test and $62.85 \%$ for the post-test (Wilcoxon test for paired sample: $\mathrm{z}=3.729, \mathrm{p}<0.001)$. There were no significant statistical differences among professions of healthcare providers for both pre- and post-tests.

Discussion: Delivering competencies-based course in Basic Principles of Complex Humanitarian Emergency for health care providers can help in the improvement of knowledge and skills for humanitarian assistance and disaster relief in Saudi Arabia, which is important for disaster preparedness augmentation in the kingdom. The next course for the same group may be recommended for achieving the level that will train them to participate in the National Disaster Assistance team.

Prehosp Disaster Med 2019;34(Suppl. 1):s105

doi:10.1017/S1049023X1900219X

\section{Behavioral Health Resource Utilization of Emergency Department Patients Presenting from Mass Gathering Events}

Dr. Charles Hebert, Dr. Gary Peksa, Dr. Joshua DeMott

Rush University Medical Center, Chicago, Ilinois, USA, Chicago, United States

Introduction: Behavioral health needs of attendees at mass gathering events who require emergency department (ED) evaluation are poorly understood. Appropriate resource allocation of mental health staff and other behavioral interventions necessary to support this patient population are also unclear.

Aim: To describe behavioral characteristics and psychiatric resource utilization of patients presenting to a tertiary academic medical center emergency department from mass gathering events.

Methods: Single-center retrospective study evaluating attendees at mass gathering events who presented to a Chicago ED. Electronic medical records for patients presenting between October 13, 2013, and December 31, 2015, were reviewed and descriptive analyses performed.

Results: 209 distinct records were reviewed. Most patients presented from large outdoor concerts $(n=186,89 \%)$. Forty-two (20.1\%) reported a mental health complaint at presentation, including concerns related to pre-existing psychiatric disturbances or onset of new symptoms. Twenty-seven of the total cohort (12.9\%) endorsed a prior psychiatric history. Thirty-five (16.7\%) reported use of prescribed psychotropic medications, including antidepressants, stimulants, mood stabilizers, and others. Diagnostic testing among the total sample included serum ethanol measurement (31.1\%), urinary toxicology (25.4\%), acetaminophen (6.2\%), aspirin (5.3\%), and creatine kinase measurements (11\%). Computed brain tomography was ordered for 20 patients (9.6\%). Twelve patients (5.7\%) 